PROCEEDINGS OF THE

AMERICAN MATHEMATICAL SOCIETY

Volume 128, Number 12, Pages 3749-3759

S 0002-9939(00)05487-3

Article electronically published on June 7, 2000

\title{
TOTALLY NONNEGATIVE AND OSCILLATORY ELEMENTS IN SEMISIMPLE GROUPS
}

\author{
SERGEY FOMIN AND ANDREI ZELEVINSKY \\ (Communicated by John R. Stembridge)
}

\begin{abstract}
We generalize the well known characterizations of totally nonnegative and oscillatory matrices, due to F. R. Gantmacher, M. G. Krein, A. Whitney, C. Loewner, M. Gasca, and J. M. Peña to the case of an arbitrary complex semisimple Lie group.
\end{abstract}

\section{INTRODUCTION}

In this note, we extend some classical theorems in the theory of total positivity to the case of an arbitrary semisimple complex Lie group. We begin by reviewing the results we are going to generalize.

Let $G=G L_{n}(\mathbb{C})$ or $S L_{n}(\mathbb{C})$. The following theorem is due to C. Loewner [10] and A. Whitney [12] (cf. [9] Lemma 9.1]).

Theorem 1.1. For a matrix $x \in G$, the following are equivalent:

(a) all minors of $x$ are nonnegative real numbers;

(b) $x$ lies in the closure of the set of matrices with positive minors;

(c) $x$ belongs to the multiplicative monoid in $G$ generated by elementary Jacobi matrices with nonnegative matrix entries.

(Here in (c), an "elementary Jacobi matrix" is a matrix that differs from the identity matrix in a single entry, located either on the main diagonal, or immediately above or below it.)

A matrix $x \in G$ satisfying any of the equivalent conditions (a)-(c) above is called totally nonnegative. Furthermore, $x$ is totally positive if all its minors are positive. Totally positive matrices are distinguished among the totally nonnegative ones as follows.

Theorem 1.2. For a totally nonnegative matrix $x \in G$, the following are equivalent:

(d) $x$ is totally positive;

(e) all solid minors of $x$ involving either $x_{1 n}$ or $x_{n 1}$ are positive;

(f) $x$ belongs to the intersection of opposite open Bruhat cells $B w_{\mathrm{o}} B \cap B_{-} w_{\mathrm{o}} B_{-}$.

Received by the editors November 18, 1998 and, in revised form, February 26, 1999.

2000 Mathematics Subject Classification. Primary 22E46; Secondary 14M15, 15A48, 20 F55.

Key words and phrases. Total positivity, oscillatory element, semisimple Lie group.

The authors were supported in part by NSF grants \#DMS-9625511 and \#DMS-9700927.

(C)2000 by Sergey Fomin and Andrei Zelevinsky 
(Here in (e), a "solid minor" is a minor formed by several consecutive rows and as many consecutive columns. In (f), we denote by $B$ (resp. $B_{-}$) the subgroup of upper-triangular (resp. lower-triangular) matrices, and $w_{\mathrm{o}}$ is the permutation matrix with 1's on the main antidiagonal.)

Part $(\mathrm{d}) \Longleftrightarrow(\mathrm{e})$ of Theorem 1.2 is a refinement of the classical Fekete criterion due to M. Gasca and J. M. Peña [8] Theorem 4.3]; the equivalence (e) $\Longleftrightarrow$ (f) is a well-known (and easy) linear-algebraic fact (cf., e.g., [6, Theorem II.4.1]).

In their pioneering study of total positivity undertaken in 1930s, F. R. Gantmacher and M. G. Krein introduced and studied the intermediate class of oscillatory matrices defined as follows: a matrix $x \in G$ is called oscillatory if $x$ is totally nonnegative while some power of $x$ is totally positive. The following characterization of this class was obtained in [7] (see §II.7; cf. also [9, Theorem 9.3]).

Theorem 1.3. For a totally nonnegative matrix $x \in G$, the following are equivalent:

(g) $x$ is oscillatory;

(h) $x_{i, i+1}>0$ and $x_{i+1, i}>0$ for $i=1, \ldots, n-1$.

Gantmacher and Krein [7] further showed that the definition of oscillatory matrices can be refined as follows.

Theorem 1.4. A totally nonnegative matrix $x \in G$ is oscillatory if and only if $x^{n-1}$ is totally positive.

In this paper, we extend Theorems 1.1, 1.3, and 1.4 to an arbitrary semisimple complex Lie group $G$, using the notion of generalized minors introduced in [4]. A generalization of Theorem 1.2 follows from results in [1] or 4], and is presented below (see Theorem 3.2) for the sake of completeness.

Even in the case of $S L_{n}$, our version of the criterion (h) is more general than the one given above. (Earlier in [4, we gave a family of total positivity criteria generalizing (e).) It should also be noted that our proofs are quite different from the ones in [8, 7, 9, 10, 12]. Our main technical tools involve combinatorics of reduced words in Weyl groups, the subdivison of a semisimple group into double Bruhat cells, and the "generalized determinantal calculus" developed in [4; in particular, the fundamental role is played by a generalized determinantal identity [4. Theorem 1.17].

The study of total positivity in reductive groups other than $G L_{n}$ and $S L_{n}$ was initiated by G. Lusztig [11], who suggested using the natural generalization of (c) as the definition of a total nonnegative element. Our extension of the equivalence (a) $\Longleftrightarrow$ (c) can be rephrased as saying that Lusztig's definition is equivalent to the one in terms of the generalized minors of [4].

\section{TERMinOLOGY AND NOTATION}

We will use the setup of [4], which is briefly reviewed below in this section. Proofs and further details can be found in [4] (see Sections 1.1-1.4).

Let $G$ be a simply connected semisimple complex Lie group of rank $r$ with a fixed pair of opposite Borel subgroups $B_{-}$and $B$; thus $H=B_{-} \cap B$ is a maximal torus in $G$. Let $N_{-}$and $N$ be the unipotent radicals of $B_{-}$and $B$, respectively.

Let $\alpha_{1}, \ldots, \alpha_{r}$ be the system of simple roots for which the corresponding root subgroups are contained in $N$. For every $i \in[1, r]$, let $\varphi_{i}: S L_{2} \rightarrow G$ be the canonical embedding corresponding to the simple root $\alpha_{i}$. 
For any nonzero $t \in \mathbb{C}$ and any $i \in[1, r]$, we define

$$
x_{\bar{i}}(t)=\varphi_{i}\left(\begin{array}{ll}
1 & 0 \\
t & 1
\end{array}\right), \quad t^{h_{i}}=\varphi_{i}\left(\begin{array}{cc}
t & 0 \\
0 & t^{-1}
\end{array}\right), \quad x_{i}(t)=\varphi_{i}\left(\begin{array}{ll}
1 & t \\
0 & 1
\end{array}\right) .
$$

Thus $t^{h_{i}} \in H$, and $t \mapsto x_{i}(t)$ (resp. $t \mapsto x_{\bar{i}}(t)$ ) is a one-parameter subgroup in $N$ (resp. in $N_{-}$).

The weight lattice $P$ can be defined as the group of multiplicative characters of $H$, here written in the exponential notation: a weight $\gamma \in P$ acts by $a \mapsto a^{\gamma}$. The lattice $P$ has a $\mathbb{Z}$-basis formed by the fundamental weights $\omega_{1}, \ldots, \omega_{r}$ defined by $\left(t^{h_{j}}\right)^{\omega_{i}}=t^{\delta_{i j}}$.

The Weyl group $W$ of $G$ is defined by $W=\operatorname{Norm}_{G}(H) / H$. The action of $W$ on $H$ by conjugation gives rise to the action of $W$ on the weight lattice $P$ given by $a^{w(\gamma)}=\left(w^{-1} a w\right)^{\gamma}$ for $w \in W, a \in H, \gamma \in P$. The group $W$ is a Coxeter group with simple reflections $s_{1}, \ldots, s_{r}$ which can be defined by specifying their representatives in $\operatorname{Norm}_{G}(H)$ : we set

$$
\overline{s_{i}}=\varphi_{i}\left(\begin{array}{cc}
0 & -1 \\
1 & 0
\end{array}\right) \in \operatorname{Norm}_{G}(H) .
$$

The family $\left\{\overline{s_{i}}\right\}$ satisfies the braid relations in $W$; thus the representative $\bar{w}$ can be unambiguously defined for any $w \in W$ by requiring that $\overline{u v}=\bar{u} \cdot \bar{v}$ whenever $\ell(u v)=\ell(u)+\ell(v)$; here $\ell(w)$ denotes the length of $w \in W$.

A reduced word for $w \in W$ is a sequence of indices $\left(i_{1}, \ldots, i_{m}\right)$ that satisfies $w=s_{i_{1}} \cdots s_{i_{m}}$ and has the shortest possible length $m=\ell(w)$. The set of reduced words for $w$ will be denoted by $R(w)$.

As customary, $w_{\mathrm{o}}$ denotes the unique element of maximal length in $W$.

We denote by $G_{0}=N_{-} H N$ the open subset of elements $x \in G$ that have Gaussian decomposition; this decomposition will be written as $x=[x]_{-}[x]_{0}[x]_{+}$.

For $u, v \in W$ and $i \in[1, r]$, the generalized minor $\Delta_{u \omega_{i}, v \omega_{i}}$ is the regular function on $G$ whose restriction to the open set $\bar{u} G_{0} \bar{v}^{-1}$ is given by

$$
\Delta_{u \omega_{i}, v \omega_{i}}(x)=\left(\left[\bar{u}^{-1} x \bar{v}\right]_{0}\right)^{\omega_{i}} .
$$

It can be shown that $\Delta_{u \omega_{i}, v \omega_{i}}$ depends on the weights $u \omega_{i}$ and $v \omega_{i}$ alone, not on the particular choice of $u$ and $v$. In the special case $G=S L_{n}$, the generalized minors are nothing but the ordinary minors of a matrix.

\section{MAin Results}

We generalize the Loewner-Whitney Theorem (Theorem 1.1) as follows.

Theorem 3.1. For an element $x \in G$, the following are equivalent:

(a) all generalized minors $\Delta_{\gamma, \delta}$ take nonnegative real values at $x$;

(b) $x$ lies in the closure of the set of elements with positive generalized minors;

(c) $x$ lies in the multiplicative monoid generated by the elements of the form $t^{h_{i}}$, $x_{i}(t)$, and $x_{\bar{i}}(t)$, with positive $t$.

An element $x \in G$ satisfying any of the equivalent conditions (a)-(c) of Theorem 3.1 is called totally nonnegative. The set of all such elements is denoted by $G \geq 0$.

The following generalization of Theorem 1.2 is immediate from the results in Lusztig [11]; a proof based on the results in [4] will be given in Section 4 below. 
Theorem 3.2. For an element $x \in G_{\geq 0}$, the following are equivalent:

(d) all generalized minors of $x$ are positive;

(e) $\Delta_{\omega_{i}, w_{\mathrm{o}} \omega_{i}}(x)>0$ and $\Delta_{w_{\mathrm{o}} \omega_{i}, \omega_{i}}(x)>0$ for any $i \in[1, r]$;

(f) $x$ belongs to the intersection of open Bruhat cells $B w_{\mathrm{o}} B \cap B_{-} w_{\mathrm{o}} B_{-}$.

An element $x \in G$ satisfying any of the equivalent conditions (d)-(f) of Theorem 3.2 is called totally positive. The set of all such elements will be denoted by $G_{>0}$.

Let us call an element $x \in G_{\geq 0}$ oscillatory if for some positive integer $m$, the element $x^{m}$ is totally positive. We will give equivalent reformulations of this property which in particular generalize the criterion (h) in Theorem 1.3 In fact, our version of criterion (h) will be more general even in the special case $G=S L_{n}$.

Let $i$ and $j$ be two indices lying in the same connected component of the Dynkin graph of $G$ (the case $j=i$ is not excluded). Let

$$
i=i(1), i(2), \ldots, i(l)=j
$$

be the unique path from $i$ to $j$ in the Dynkin graph. Thus $\{i(k), i(k+1)\}$ is an edge for $k=1, \ldots, l-1$, and all indices $i(k)$ are distinct. Let us denote $c(j \rightarrow i)=$ $s_{i(2)} s_{i(3)} \cdots s_{j}($ in particular, $c(i \rightarrow i)=e)$, and set

$$
\begin{aligned}
& \Delta_{j \rightarrow i}=\Delta_{c(j \rightarrow i) \omega_{j}, s_{i} c(j \rightarrow i) \omega_{j}}, \\
& \Delta_{j \rightarrow \bar{i}}=\Delta_{s_{i} c(j \rightarrow i) \omega_{j}, c(j \rightarrow i) \omega_{j}} .
\end{aligned}
$$

For a given $i$, each minor of the form $\Delta_{j \rightarrow i}$ (resp. $\left.\Delta_{j \rightarrow i}\right)$ is called an $i$-indicator (resp. $\bar{i}$-indicator). (This terminology is justified by Lemma 6.3 below.)

Theorem 3.3. Let $C$ be a collection of $2 r$ generalized minors that contains, for every $i \in[1, r]$, an $i$-indicator and an $\bar{i}$-indicator. Then, for an element $x \in G_{\geq 0}$, the following are equivalent:

(g) $x$ is oscillatory;

(h) $\Delta(x)>0$ for any $\Delta \in C$;

(i) $x$ does not belong to a proper parabolic subgroup of $G$ containing $B$ or $B_{-}$.

Note that the equivalence $(\mathrm{g}) \Longleftrightarrow(\mathrm{h})$ in Theorem 3.3 generalizes Theorem 1.3 Indeed, for $G=S L_{n}$ and the standard numbering of fundamental weights, one checks that $x_{i, i+1}=\Delta_{1 \rightarrow i}$ and $x_{i+1, i}=\Delta_{1 \rightarrow \bar{i}}$. Thus the set $C$ consisting of these matrix entries satisfies the condition of Theorem 3.3

Our last main result is a generalization of Theorem 1.4 to all semisimple groups.

Theorem 3.4. For any given $G$, there exists a positive integer $m$ with the following property: an element $x \in G_{\geq 0}$ is oscillatory if and only if $x^{m} \in G_{>0}$. A positive integer $m$ has this property if and only if for any permutation $\mathbf{i}=\left(i_{1}, \ldots, i_{r}\right)$ of indices $1, \ldots, r$, the concatenation of $m$ copies of $\mathbf{i}$ has a reduced word for $w_{\mathrm{o}}$ as a subword.

Let $m(G)$ denote the smallest positive integer $m$ that has the property described in Theorem 3.4.

Theorem 3.5. For a simple group $G$, the value of $m(G)$ is given by the table

\begin{tabular}{|c|c|c|c|c|c|c|c|c|c|}
\hline Type & $A_{r}$ & $B_{r}$ or $C_{r}$ & $D_{r}, r$ even & $D_{r}, r$ odd & $E_{6}$ & $E_{7}$ & $E_{8}$ & $F_{4}$ & $G_{2}$ \\
\hline$m(G)$ & $r$ & $r$ & $r-1$ & $r$ & 8 & 9 & 15 & 6 & 3 \\
\hline
\end{tabular}

The remaining sections contain the proofs of Theorems 3.13 .5 . 


\section{Proof of Theorem 3.2}

The group $G$ has two Bruhat decompositions, with respect to opposite Borel subgroups $B$ and $B_{-}$:

$$
G=\bigcup_{u \in W} B u B=\bigcup_{v \in W} B_{-} v B_{-} .
$$

The double Bruhat cells $G^{u, v}$ are defined by $G^{u, v}=B u B \cap B_{-} v B_{-}$.

Let $H_{>0}$ be the subgroup of $H$ generated by the elements $t^{h_{i}}$ for any $t>0$ and $i \in[1, r]$; equivalently, $H_{>0}$ consists of all $a \in H$ such that $a^{\gamma}>0$ for any weight $\gamma \in P$. Following G. Lusztig, let us define the set $G_{\geq 0}$ as the multiplicative monoid in $G$ generated by $H_{>0}$ and the elements $x_{i}(t)$ and $x_{\bar{i}}(t)$, for $i \in[1, r]$ and $t>0$. In other words, we use condition (c) of Theorem 3.1 as the interim definition of $G_{\geq 0}$.

The set $G_{\geq 0}$ is the disjoint union of totally positive varieties $G_{>0}^{u, v}$ defined by

$$
G_{>0}^{u, v}=G_{\geq 0} \cap G^{u, v} .
$$

We denote $[\overline{1}, \bar{r}]=\{\overline{1}, \ldots, \bar{r}\}$. For any sequence $\mathbf{i}=\left(i_{1}, \ldots, i_{m}\right)$ of indices from the alphabet $[1, r] \cup[\overline{1}, \bar{r}]$, let us define the map $x_{\mathbf{i}}: H \times \mathbb{C}^{m} \rightarrow G$ by

$$
x_{\mathbf{i}}\left(a ; t_{1}, \ldots, t_{m}\right)=a x_{i_{1}}\left(t_{1}\right) \cdots x_{i_{m}}\left(t_{m}\right) .
$$

By definition, an element $x \in G_{\geq 0}$ can be represented as $x=x_{\mathbf{i}}\left(a ; t_{1}, \ldots, t_{m}\right)$, for some sequence $\mathbf{i}$, with all the $t_{k}$ positive and $a \in H_{>0}$.

A double reduced word for the elements $u, v \in W$ is a reduced word for an element $(u, v)$ of the Coxeter group $W \times W$. To avoid confusion, we will use the indices $\overline{1}, \overline{2}, \ldots, \bar{r}$ for the simple reflections in the first copy of $W$, and $1,2, \ldots, r$ for the second copy. A double reduced word for $(u, v)$ is nothing but a shuffle of a reduced word for $u$ written in the alphabet $[\overline{1}, \bar{r}]$ and a reduced word for $v$ written in the alphabet $[1, r]$. We denote the set of double reduced words for $(u, v)$ by $R(u, v)$.

The weak order is the partial order on $W$ defined as follows: $u^{\prime} \preceq u$ stands for $\ell(u)=\ell\left(u^{\prime}\right)+\ell\left(u^{\prime-1} u\right)$. (In other words, a reduced word for $u^{\prime}$ can be extended on the right to form a reduced word for $u$.) We note that $w \preceq w_{\text {o }}$ for any $w \in W$.

The following lemma provides alternative descriptions of the totally positive varieties $G_{>0}^{u, v}$.

Lemma 4.1. For an element $x \in G^{u, v}$, the following conditions are equivalent:

$\left(\mathrm{c}^{\prime}\right) x \in G_{>0}^{u, v}$;

$\left(\mathrm{c}^{\prime \prime}\right)$ for some (equivalently, any) double reduced word $\mathbf{i} \in R(u, v)$, we have $x=$ $x_{\mathbf{i}}\left(a ; t_{1}, \ldots, t_{m}\right)$ with $a \in H_{>0}$ and $t_{1}, \ldots, t_{m}>0$;

$\left(\mathrm{c}^{\prime \prime \prime}\right) \Delta_{u^{\prime} \omega_{i}, v^{\prime} \omega_{i}}(x)>0$ for all $i \in[1, r]$ and all $u^{\prime} \preceq u, v^{\prime} \preceq v^{-1}$.

Proof. See [4, Theorems 1.3 and 1.11]. (The equivalence $\left(\mathrm{c}^{\prime}\right) \Longleftrightarrow\left(\mathrm{c}^{\prime \prime}\right)$ was essentially established in [11.)

Now everything is ready for the proof of Theorem 3.2 The implication (f) $\Longrightarrow(\mathrm{d})$ is a special case of $\left(\mathrm{c}^{\prime}\right) \Longrightarrow\left(\mathrm{c}^{\prime \prime \prime}\right)$, while $(\mathrm{d}) \Longrightarrow(\mathrm{e})$ is trivial. Finally, to show that (e) $\Longleftrightarrow(\mathrm{f})$, it suffices to note that

$$
G^{w_{\mathrm{o}}, w_{\mathrm{o}}}=w_{\mathrm{o}} G_{0} \cap G_{0} w_{\mathrm{o}}=\left\{x \in G: \Delta_{w_{\mathrm{o}} \omega_{i}, \omega_{i}}(x) \neq 0, \Delta_{\omega_{i}, w_{\mathrm{o}} \omega_{i}}(x) \neq 0(i \in[1, r])\right\}
$$

(cf. [4, Corollary 2.5] or [5, Proposition 4.1]). 


\section{Proof of Theorem 3.1}

5.1. Proof of $(\mathbf{b}) \Rightarrow(\mathbf{a})$. This is obvious since all generalized minors are continuous functions on $G$.

5.2. Proof of $(\mathbf{c}) \Rightarrow(\mathbf{b})$. In view of Lemma 4.1, it suffices to show that the closure of $G_{>0}^{w_{\mathrm{o}}, w_{\mathrm{o}}}$ contains all totally positive varieties $G_{>0}^{u, v}$. Suppose $x \in G_{>0}^{u, v}$ for some $u$ and $v$. Take any $\mathbf{i} \in R(u, v)$ and write $x=x_{\mathbf{i}}\left(a ; t_{1}, \ldots, t_{m}\right)$ as in $\left(\mathrm{c}^{\prime \prime}\right)$. Choose a word $\mathbf{j} \in R\left(w_{\mathrm{o}}, w_{\mathrm{o}}\right)$ that has $\mathbf{i}$ as an initial segment. Then

$$
x=x_{\mathbf{i}}\left(a ; t_{1}, \ldots, t_{m}\right)=\lim _{t \rightarrow+0} x_{\mathbf{j}}\left(a ; t_{1}, \ldots, t_{m}, t, \ldots, t\right),
$$

and (b) follows.

5.3. Proof of $(\mathbf{a}) \Rightarrow(\mathbf{c})$. Suppose that $x \in G^{u, v}$ satisfies (a). It suffices to check condition $\left(\mathrm{c}^{\prime \prime \prime}\right)$ in Lemma 4.1. Let $\Sigma(x)$ denote the set of all pairs $\left(u^{\prime}, v^{\prime}\right) \in W \times W$ such that $\Delta_{u^{\prime} \omega_{i}, v^{\prime} \omega_{i}}(x)>0$ for all $i$. Our aim is to show that

$$
\left(u^{\prime}, v^{\prime}\right) \in \Sigma(x) \text { for } u^{\prime} \preceq u, v^{\prime} \preceq v^{-1} .
$$

As a first step we notice that

$$
(u, e),\left(e, v^{-1}\right) \in \Sigma(x)
$$

this follows from the well-known fact that $\Delta_{u \omega_{i}, \omega_{i}}$ vanishes nowhere on the Bruhat cell BuB; see, e.g., [5, Lemma 3.4].

We shall write $u^{\prime} \rightarrow u^{\prime \prime}$ if $u^{\prime \prime}=u^{\prime} s_{i}$ for some $i$, and $\ell\left(u^{\prime \prime}\right)=\ell\left(u^{\prime}\right)+1$. In view of (5.3), the desired inclusions (5.2) are consequences of the following statements:

$$
\begin{aligned}
& \text { if } u^{\prime} \rightarrow u^{\prime \prime} \text { and }\left(u^{\prime \prime}, e\right) \in \Sigma(x) \text {, then }\left(u^{\prime}, e\right) \in \Sigma(x) \text {; } \\
& \text { if } u^{\prime} \rightarrow u^{\prime \prime} \text { and }\left(e, u^{\prime \prime}\right) \in \Sigma(x) \text {, then }\left(e, u^{\prime}\right) \in \Sigma(x) \text {; }
\end{aligned}
$$

Our proof of both (5.4) and (5.5) relies on the following identity [4, Theorem 1.17]:

$$
\Delta_{u^{\prime} \omega_{i}, v^{\prime} \omega_{i}} \Delta_{u^{\prime \prime} \omega_{i}, v^{\prime \prime} \omega_{i}}=\Delta_{u^{\prime} \omega_{i}, v^{\prime \prime} \omega_{i}} \Delta_{u^{\prime \prime} \omega_{i}, v^{\prime} \omega_{i}}+\prod_{j \neq i} \Delta_{u^{\prime} \omega_{j}, v^{\prime} \omega_{j}}^{-a_{j i}},
$$

whenever $u^{\prime} \rightarrow u^{\prime \prime}=u^{\prime} s_{i}$ and $v^{\prime} \rightarrow v^{\prime \prime}=v^{\prime} s_{i}$; here the numbers $a_{j i}$ are the entries of the Cartan matrix of $G$.

To prove (5.4), suppose that $u^{\prime} \rightarrow u^{\prime \prime}=u^{\prime} s_{i}$ and $\left(u^{\prime \prime}, e\right) \in \Sigma(x)$. Now specialize (5.6) at $v^{\prime}=e$ and evaluate both sides at $x$. Using the fact that $u^{\prime} \omega_{j}=u^{\prime \prime} \omega_{j}$ for $j \neq i$, we see that the second summand on the right-hand side is strictly positive. Since all generalized minors of $x$ are nonnegative, we conclude that both factors on the left-hand side are positive. In particular, $\Delta_{u^{\prime} \omega_{i}, \omega_{i}}(x)>0$, i.e., $\left(u^{\prime}, e\right) \in \Sigma(x)$, as desired. The second part of (5.4) is proved in the same way.

To prove (5.5), suppose that $u^{\prime} \rightarrow u^{\prime \prime}=u^{\prime} s_{i}$ and $v^{\prime} \rightarrow v^{\prime \prime}=v^{\prime} s_{j}$, and both $\left(u^{\prime}, v^{\prime \prime}\right)$ and $\left(u^{\prime \prime}, v^{\prime}\right)$ belong to $\Sigma(x)$. We need to show that $\Delta_{u^{\prime \prime} \omega_{k}, v^{\prime \prime} \omega_{k}}(x)>0$ for all $k$. If $k \neq i$, then $u^{\prime \prime} \omega_{k}=u^{\prime} \omega_{k}$ and we are done since $\left(u^{\prime}, v^{\prime \prime}\right) \in \Sigma(x)$. The case $k \neq j$ is treated in the same way. It thus remains to consider the case $k=j=i$. But then in (5.6), the first summand on the right (evaluated at $x$ ) is positive, implying $\Delta_{u^{\prime \prime} \omega_{i}, v^{\prime \prime} \omega_{i}}(x)>0$, as desired. This completes the proof of Theorem 3.1 


\section{Proof of Theorem 3.3}

6.1. Proof of $(\mathbf{g}) \Rightarrow(\mathbf{i})$. Since total positivity is described by condition (f) in Theorem [3.2, it suffices to show that every proper parabolic subgroup of $G$ containing $B$ or $B_{-}$has empty intersection with the open double Bruhat cell $G^{w_{\circ}, w_{\circ}}$. The latter follows at once from the well known description of maximal proper parabolic subgroups containing $B$ or $B_{-}$: they are the subgroups $P_{1}, \ldots, P_{r}$ and $P_{\overline{1}}, \ldots, P_{\bar{r}}$ given by

$$
P_{i}=\bigcup_{i \notin \operatorname{Supp}(v)} B_{-} v B_{-}, \quad P_{\bar{i}}=\bigcup_{i \notin \operatorname{Supp}(u)} B u B
$$

where $\operatorname{Supp}(w)$ denotes the set of indices that occur in some (equivalently, any) reduced word for $w \in W$.

6.2. Proof of $(\mathbf{i}) \Rightarrow(\mathbf{g})$. Consider the monoid $\mathcal{H}$ whose generators $T_{1}, \ldots, T_{r}$ are subject to relations

$$
\begin{aligned}
T_{i}^{2} & =T_{i} ; \\
\underbrace{T_{i} T_{j} T_{i} \cdots}_{m_{i j}} & =\underbrace{T_{j} T_{i} T_{j} \cdots}_{m_{i j}}(i \neq j) ;
\end{aligned}
$$

here $m_{i j}$ is the order of $s_{i} s_{j}$ in $W$. A well known theorem of Tits on reduced words (see, e.g., [3, II, $\S 3 \mathrm{C}]$ ) has the following implications. First, if $\left(i_{1}, \ldots, i_{m}\right) \in$ $R(w)$, then the product $T_{i_{1}} \cdots T_{i_{m}}$ only depends on $w$ and so can be unambiguously denoted by $T_{w}$. Second, the correspondence $w \mapsto T_{w}$ is a bijection between $W$ and $\mathcal{H}$. Finally, we have the following criterion for determining when a product of generators is equal to $T_{w_{\mathrm{o}}}$.

Lemma 6.1. For a word $\left(i_{1}, \ldots, i_{N}\right)$ in the alphabet $[1, r]$, we have $T_{i_{1}} \cdots T_{i_{N}}=T_{w_{\mathrm{o}}}$ if and only if this word has a reduced word for $w_{\mathrm{o}}$ as a subword.

The relevance of $\mathcal{H}$ to our problem is clear from the following lemma.

Lemma 6.2. For any $x \in G_{>0}^{u, v}$ and $y \in G_{>0}^{u^{\prime}, v^{\prime}}$, we have $x y \in G_{>0}^{u^{\prime \prime}, v^{\prime \prime}}$, where the elements $u^{\prime \prime}$ and $v^{\prime \prime}$ are given by $T_{u^{\prime \prime}}=T_{u} T_{u^{\prime}}$ and $T_{v^{\prime \prime}}=T_{v} T_{v^{\prime}}$.

Proof. The proof follows from condition $\left(\mathrm{c}^{\prime \prime}\right)$ of Lemma 4.1, together with the commutation relations among the elementary factors $x_{i}(t)$ and $x_{\bar{i}}(t)$, as given in [1] Theorem 3.1] and [4 Section 2.2].

By Lemma 6.2 and condition (f) of Theorem [3.2, for any $x \in G_{>0}^{u, v}$ and any positive integer $m$, we have

$$
x^{m} \in G_{>0} \Leftrightarrow T_{u}^{m}=T_{v}^{m}=T_{w_{\mathrm{o}}} .
$$

Suppose that a totally nonnegative element $x$ satisfies condition (i). By (6.1), $x \in G_{>0}^{u, v}$ for some elements $u, v \in W$ such that $\operatorname{Supp}(u)=\operatorname{Supp}(v)=[1, r]$. We need to show that $x$ is oscillatory. In view of (6.2), this means that $T_{u}^{m}=T_{v}^{m}=T_{w_{\text {o }}}$ for sufficiently large $m$. The latter is clear from Lemma 6.1: just take $m=\ell\left(w_{\mathrm{o}}\right)$. 
6.3. Proof of $(\mathbf{h}) \Leftrightarrow(\mathbf{i})$. This equivalence can be restated as follows.

Lemma 6.3. Let $i \in[1, r]$, and let $\Delta$ be an $i$-indicator (resp. $\bar{i}$-indicator). Then $\Delta$ vanishes on $P_{i}$ (resp. $P_{\bar{i}}$ ), and $\Delta(x)>0$ for any $x \in G_{\geq 0}$ outside $P_{i}$ (resp. $P_{\bar{i}}$ ).

Proof. It is enough to consider $i$-indicators, the case of $\bar{i}$-indicators being totally similar. Changing if necessary the numeration of fundamental weights, we can assume without loss of generality that $i=1$, and

$$
\Delta=\Delta_{j \rightarrow 1}=\Delta_{u \omega_{j}, s_{1} u \omega_{j}},
$$

where $u=c(j \rightarrow 1)=s_{2} \cdots s_{j}$, with nonzero Cartan matrix entries $a_{k, k+1}$ for $k=1, \ldots, j-1$.

First let us show that $\Delta(x)=0$ for $x \in P_{1}$. We will denote by $x^{T}$ the "transpose" of $x$; more precisely, $x \mapsto x^{T}$ is the anti-automorphism of $G$ defined by

$$
a^{T}=a \quad(a \in H), \quad x_{i}(t)^{T}=x_{\bar{i}}(t), \quad x_{\bar{i}}(t)^{T}=x_{i}(t) .
$$

As in 4, we will use the notation $\Delta^{\omega_{i}}=\Delta_{\omega_{i}, \omega_{i}}$ for the $i$ th "principal minor." Using [4, (1.10), (2.25)], we obtain:

$$
\begin{aligned}
& \Delta(x)=\Delta_{u \omega_{j}, s_{1} u \omega_{j}}(x)=\Delta_{s_{1} u \omega_{j}, u \omega_{j}}\left(x^{T}\right)=\Delta^{\omega_{j}}\left(\overline{s_{1} u}-1 x^{T} \bar{u}\right) \\
& =\Delta^{\omega_{j}}\left({\overline{u^{-1} s_{1} u}}^{-1} \overline{u^{-1}} x^{T} \bar{u}\right)=\Delta_{u^{-1} s_{1} u \omega_{j}, \omega_{j}}\left(\overline{u^{-1}} x^{T} \bar{u}\right) .
\end{aligned}
$$

Observe that $\overline{u^{-1}} x^{T} \bar{u} \in P_{\overline{1}}$ for any $x \in P_{1}$ (since all three factors belong to $P_{\overline{1}}$ ). It remains to prove that $\Delta_{u^{-1}} s_{1} u \omega_{j}, \omega_{j}$ vanishes on $P_{\overline{1}}$. To see this, we use the following description of $P_{\overline{1}}$ equivalent to (6.1): $P_{\overline{1}}=\pi^{-1}\left(X_{w_{0}^{\prime}}\right)$, where $\pi$ is the projection of $G$ onto the flag variety $G / B$, the element $w_{\mathrm{o}}^{\prime} \in W$ is the longest element of the parabolic subgroup generated by $s_{2}, \ldots, s_{r}$, and $X_{w}$ is the Schubert variety corresponding to $w$ (i.e., the closure of the Schubert cell $(B w B) / B$ ). The vanishing of $\Delta_{u^{-1}} s_{1} u \omega_{j}, \omega_{j}$ on $P_{\overline{1}}$ is equivalent to the vanishing of the Plücker coordinate $p_{\gamma}$ corresponding to the weight $\gamma=u^{-1} s_{1} u \omega_{j}$ on the $X_{w_{\mathrm{o}}^{\prime}}$. Recall (see, e.g., [5] Lemma $3.4])$ that, in general, a Plücker coordinate $p_{\delta}$ vanishes on a Schubert variety $X_{w}$ if and only if $v \not \leq w$ in the Bruhat order, where $v \in W$ is the element of minimal length satisfying $\delta=v \omega_{j}$. The claim now follows by applying this criterion to $v=u^{-1} s_{1} u$ and $w=w_{\mathrm{o}}^{\prime}$; the condition $u^{-1} s_{1} u \not \leq w_{\mathrm{o}}^{\prime}$ is satisfied since $1 \in \operatorname{Supp}\left(u^{-1} s_{1} u\right)$.

To complete the proof of Lemma 6.3 and Theorem 3.3 it remains to show that $\Delta_{j \rightarrow 1}(x)>0$ for any element $x \in G_{\geq 0}$ not belonging to $P_{1}$. We proceed by induction on $j$. Let us first consider the case $j=1$ when we need to show that $\Delta_{\omega_{1}, s_{1} \omega_{1}}(x)>0$. Since $\Delta_{\omega_{1}, s_{1} \omega_{1}}\left(b_{-} x\right)=\Delta^{\omega_{1}}\left(b_{-}\right) \Delta_{\omega_{1}, s_{1} \omega_{1}}(x)$ for any $b_{-} \in B_{-}$, we can assume without loss of generality that $x$ has the form

$$
x=x_{i_{1}}\left(t_{1}\right) \cdots x_{i_{m}}\left(t_{m}\right)
$$

for some sequence of (unbarred) indices $i_{1}, \ldots, i_{m}$ and some positive numbers $t_{1}, \ldots, t_{m}$. The condition $x \notin P_{1}$ means that at least one of the indices $i_{k}$ is equal to 1 ; let $k$ be the maximal index such that $i_{k}=1$. Using the fact that $\overline{s_{1}}-1$ for any $i \neq 1$, and the commutation relation $x_{i}(t) \overline{s_{i}}=x_{\bar{i}}\left(t^{-1}\right) t^{h_{i}} x_{i}\left(-t^{-1}\right)$ (see [4] (2.13)]), we conclude that

$$
\begin{array}{r}
\Delta_{\omega_{1}, s_{1} \omega_{1}}(x)=\Delta^{\omega_{1}}\left(x_{i_{1}}\left(t_{1}\right) \cdots x_{i_{k}}\left(t_{k}\right) \overline{s_{1}} \cdot\left(\overline{s_{1}}{ }^{-1} x_{i_{k+1}}\left(t_{k+1}\right) \cdots x_{i_{m}}\left(t_{m}\right) \overline{s_{1}}\right)\right) \\
=\Delta^{\omega_{1}}\left(x_{i_{1}}\left(t_{1}\right) \cdots x_{i_{k}}\left(t_{k}\right) \overline{s_{1}}\right)=\Delta^{\omega_{1}}\left(x_{i_{1}}\left(t_{1}\right) \cdots x_{i_{k-1}}\left(t_{k-1}\right) x_{\overline{1}}\left(t_{k}^{-1}\right) t_{k}^{h_{1}}\right) .
\end{array}
$$


Since the element $x^{\prime}=x_{i_{1}}\left(t_{1}\right) \cdots x_{i_{k-1}}\left(t_{k-1}\right) x_{\overline{1}}\left(t_{k}^{-1}\right) t_{k}^{h_{1}}$ is totally nonnegative, and any principal minor is positive on $G_{\geq 0}$ (see [4, Corollary 2.5 and Proposition 2.29]), we conclude that $\Delta^{\omega_{1}}\left(x^{\prime}\right)>0$, as desired.

Now assume that $j \geq 2$, and that we already know that $\Delta_{j^{\prime} \rightarrow 1}(x)>0$ for $j^{\prime}=1, \ldots, j-1$. Let us apply the identity (5.6) for $i=j, u^{\prime}=s_{2} \cdots s_{j-1}$, and $v^{\prime}=s_{1} \cdots s_{j-1}$. In our present notation, it takes the following form:

$$
\Delta^{\omega_{j}} \Delta_{j \rightarrow 1}=\Delta_{u^{\prime} \omega_{j}, v^{\prime \prime} \omega_{j}} \Delta_{u^{\prime \prime} \omega_{j}, v^{\prime} \omega_{j}}+\prod_{j^{\prime}>j}\left(\Delta^{\omega_{j^{\prime}}}\right)^{-a_{j^{\prime} j}} \cdot \prod_{j^{\prime}=1}^{j-1} \Delta_{j^{\prime} \rightarrow 1}^{-a_{j^{\prime} j}} ;
$$

here we used that $\Delta_{u \omega_{j}, v \omega_{j}}=\Delta^{\omega_{j}}$ whenever $u$ and $v$ belong to the parabolic subgroup of $W$ generated by all simple reflections except $s_{j}$. By the induction hypothesis, the second summand in the right-hand side of (6.3) is positive at $x$, while the first one is nonnegative. Hence $\Delta_{j \rightarrow 1}(x)>0$, completing the proof.

\section{Proof of Theorems 3.4 and 3.5}

7.1. Proof of Theorem [3.4, This is an immediate consequence of (6.2) and Lemma 6.1

7.2. Proof of Theorem 3.5. We will need some basic facts about Coxeter elements in Weyl groups (the proofs can be found in 2, Section V.6]). Recall that a Coxeter element $c \in W$ is a product of simple reflections $s_{1}, \ldots, s_{r}$ taken in any order. All such elements are conjugate to each other and thus have the same order; this order is called the Coxeter number of $W$ and denoted by $h$. Here are the statements we need:

(C1) If $W$ is irreducible, then $h / 2=\ell\left(w_{\mathrm{o}}\right) / r$.

(C2) If $w_{\mathrm{o}}=-1$ (i.e., $w_{\mathrm{o}}(\lambda)=-\lambda$ for any weight $\lambda$ ), then $h$ is even, and $c^{h / 2}=w_{\mathrm{o}}$ for any Coxeter element $c \in W$.

Now suppose that $G$ is simple, so the Weyl group $W$ is irreducible. Combining Theorem 3.4 with $(\mathrm{C} 1)-(\mathrm{C} 2)$, we conclude that $m(G)=h / 2=\ell\left(w_{\mathrm{o}}\right) / r$ whenever $w_{\mathrm{o}}=-1$. According to the tables in 2], this gives the desired answer for $m(G)$ for all the types except $A_{r}(r \geq 2), D_{r}$ ( $r$ odd), and $E_{6}$. Let us consider these remaining cases separately.

Throughout, we denote by $\mathbf{i}=\left(i_{1}, \ldots, i_{r}\right)$ a permutation of indices $1, \ldots, r$. It will be convenient to use the notation $\mathbf{i}^{k}$ for the concatenation of $k$ copies of $\mathbf{i}$.

Type $A_{r}$. As usual, we identify $W$ with the symmetric group $S_{r+1}$; under this identification, $s_{i}$ becomes the transposition of adjacent indices $i$ and $i+1$, and $w_{\mathrm{o}}(i)=r+2-i$ for $i \in[1, r+1]$. If $\mathbf{i}=(1, \ldots, r)$, then $\mathbf{i}^{r-1}$ does not contain a reduced word for $w_{\mathrm{o}}$, since any such reduced word must have a subword $r, r-$ $1, \ldots, 2,1$ (because $w_{\mathrm{o}}$ switches 1 and $r+1$ ). For an arbitrary permutation $\mathbf{i}$ of $1, \ldots, r$, let us now consider the sequence $\mathbf{i}^{r}$. We will form a subsequence $\mathbf{j}$ of $\mathbf{i}^{r}$ as follows. First, $\mathbf{j}$ will include all $r$ entries of $\mathbf{i}^{r}$ which are equal to 1 . Between any two consecutive 1's, there is a 2 ; let $\mathbf{j}$ include all these 2's (there will be $r-1$ of them). We then include in $\mathbf{j}$ the 3 's that interlace these 2's $(r-2$ more entries), etc. It is straightforward to check that the subsequence $\mathbf{j}$ thus obtained will be a reduced word for $w_{\mathrm{o}}$. Thus $m(G)=r$, as claimed.

Type $D_{r}\left(\begin{array}{r}r \\ \text { odd }\end{array}\right)$. In this case $h / 2=\ell\left(w_{\mathrm{o}}\right) / r=r-1$. Using the standard combinatorial interpretation of $D_{r}$, one checks that $\left(s_{1} \cdots s_{r}\right)^{r-1} \neq w_{\mathrm{o}}$, and so $m(G) \geq r$. To prove the reverse inequality, consider the standard embedding of $W$ 
into the Coxeter group $\widetilde{W}$ of type $D_{r+1}$. We know that the Coxeter number $\tilde{h}$ of $\widetilde{W}$ is equal to $2 r$, and the longest element $\tilde{w}_{\mathrm{o}} \in \widetilde{W}$ is equal to -1 . Let $\mathbf{i}=\left(i_{1}, \ldots, i_{r}\right)$ be a permutation of $1, \ldots, r$, and denote $\tilde{\mathbf{i}}=\left(i_{1}, \ldots, i_{r}, r+1\right)$. Then $\tilde{\mathbf{i}}^{r}$ is a reduced word for $\tilde{w}_{\mathrm{o}}$, and therefore it contains a reduced word for $w_{\mathrm{o}} \in W$ as a subword. We conclude that $m(G)=r$, as desired.

Type $E_{6}$. First, we verify that, in the notation of Figure 1, $\left(s_{1} s_{4} s_{6} s_{2} s_{3} s_{5}\right)^{6}=1$. Second, Lemma 6.1]implies that whenever $\left(T_{c}\right)^{k}=T_{w_{\mathrm{o}}}$ for a Coxeter element $c \in W$, then $\left(T_{c^{\prime}}\right)^{k+1}=T_{w_{\mathrm{o}}}$ for any Coxeter element $c^{\prime}$ obtained by a cyclic permutation of any reduced word for $c$. To prove the upper bound $m(G) \leq 8$, it remains to show that any permutation $\sigma$ of $1, \ldots, 6$ can be obtained from 146235 using any number of "2-moves" $\cdots i j \cdots \leadsto \cdots j i$ (for commuting $s_{i}$ and $s_{j}$ ), together with at most two cyclic rearrangements. This is done as follows. We first cyclically permute the entries of $\sigma$ to obtain a sequence that begins with 4. Any such sequence can be transformed by 2-moves into one of the four sequences 412356, 412365, 421356, 421365; further applying 2-moves, we obtain, respectively, 412356, 416235, 423516, 462351. A single cyclic permutation transforms each of these four into 641235 or 416235 or 164235 or 146235 , all of which are 2 -move-equivalent to 146235 , as desired.

The lower bound $m(G) \geq 8$ is proved by exhibiting a Coxeter element (namely, $\left.c=s_{1} s_{2} s_{3} s_{4} s_{5} s_{6}\right)$ with $\left(T_{c}\right)^{7} \neq T_{w_{\mathrm{o}}}$. (The latter verification is due to H. Derksen.)

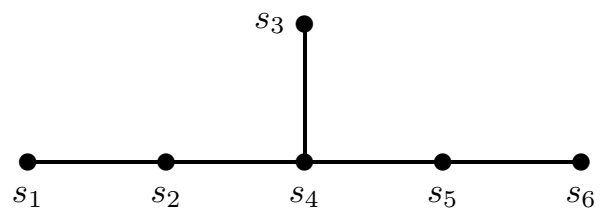

FiguRE 1. Generators of the Weyl group of type $E_{6}$

\section{ACKNOWLEDGEMENTS}

Harm Derksen contributed to Section 7.2 by first bringing the statements (C1)(C2) to our attention, and then by verifying the type $E_{6}$ case of Theorem 3.5. We are grateful to Harm for his input. We also thank the anonymous referee for helpful criticisms.

\section{REFERENCES}

1. A. Berenstein and A. Zelevinsky, Total positivity in Schubert varieties, Comment. Math. Helv. 72 (1997), 128-166. MR 99g:14064

2. N. Bourbaki, Groupes et algèbres de Lie, Ch. IV-VI, Hermann, Paris, 1968. MR 39:1590]

3. K. S. Brown, Buildings, Springer-Verlag, New York-Berlin, 1989. MR 90e:20001

4. S. Fomin and A. Zelevinsky, Double Bruhat cells and total positivity, J. Amer. Math. Soc. 12 (1999), 335-380. CMP 99:08

5. S. Fomin and A. Zelevinsky, Recognizing Schubert cells, preprint math.CO/9807079, July 1998, to appear in J. Algebr. Combinatorics.

6. F. R. Gantmacher, The theory of matrices, Chelsea Pub. Co., 1959. (Russian fourth edition: Moscow, 1988.) MR 21:6372c

7. F. R. Gantmacher and M. G. Krein, Oszillationsmatrizen, Oszillationskerne und Kleine Schwingungen Mechanischer Systeme, Akademie-Verlag, Berlin, 1960. MR 22:5161 
8. M. Gasca and J. M. Peña, Total positivity and Neville elimination, Linear Algebra Appl. 165 (1992), 25-44. MR 93d:15031

9. S. Karlin, Total positivity, Stanford University Press, 1968. MR 37:5667

10. C. Loewner, On totally positive matrices, Math. Z. 63 (1955), 338-340. MR 17:466f

11. G. Lusztig, Total positivity in reductive groups, in: Lie theory and geometry: in honor of Bertram Kostant, Progress in Mathematics 123, Birkhäuser, 1994. MR 96m:20071

12. A. M. Whitney, A reduction theorem for totally positive matrices, J. d'Analyse Math. 2 (1952), 88-92. MR 14:732c

Department of Mathematics, Massachusetts Institute of Technology, Cambridge, MASSACHUSETTS 02139

E-mail address: fomin@math.mit.edu

Current address: Department of Mathematics, University of Michigan, Ann Arbor, Michigan 48109

E-mail address: fomin@math.lsa.umich.edu

Department of Mathematics, Northeastern University, Boston, Massachusetts 02115

E-mail address: andrei@neu.edu 